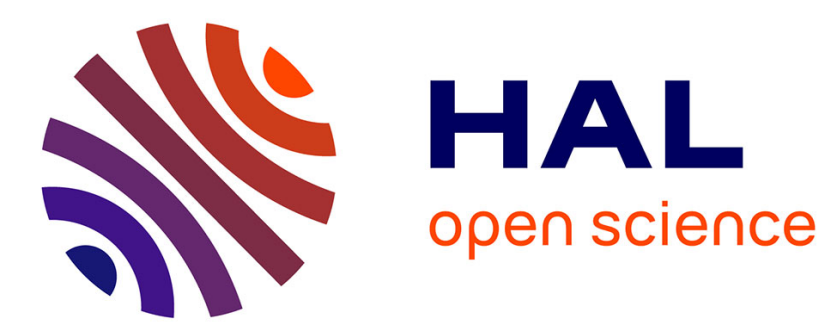

\title{
Réalisation et mise au point d'un spectromètre compton $\mathrm{Ge}(\mathrm{Li})$
}

\author{
M. Oria, R. Ripon, J. Lepetit
}

\section{To cite this version:}

M. Oria, R. Ripon, J. Lepetit. Réalisation et mise au point d'un spectromètre compton Ge(Li). Revue de Physique Appliquée, 1969, 4 (2), pp.267-268. 10.1051/rphysap:0196900402026700 . jpa-00243254

\section{HAL Id: jpa-00243254 https://hal.science/jpa-00243254}

Submitted on 1 Jan 1969

HAL is a multi-disciplinary open access archive for the deposit and dissemination of scientific research documents, whether they are published or not. The documents may come from teaching and research institutions in France or abroad, or from public or private research centers.
L'archive ouverte pluridisciplinaire HAL, est destinée au dépôt et à la diffusion de documents scientifiques de niveau recherche, publiés ou non, émanant des établissements d'enseignement et de recherche français ou étrangers, des laboratoires publics ou privés. 


\title{
RÉALISATION ET MISE AU POINT D’UN SPEGTROMÈTRE COMPTON Ge(Li)
}

\author{
M. ORIA, R. RIPON et J. LEPETIT, \\ Institut National des Sciences et Techniques Nucléaires, Saclay.
}

\begin{abstract}
Résumé. - On décrit et on expérimente un spectromètre Compton utilisant un cristal unique $\mathrm{Ge}(\mathrm{Li})$. tested.

Abstract. - A single crystal Ge(Li) Compton spectrometer is described and has been
\end{abstract}

Un spectromètre Compton - utilisant une double diode $\mathrm{Ge}(\mathrm{Li})$ - permet d'éliminer en grande partie les impulsions dues aux électrons Compton associés aux photons gamma diffusant hors du cristal [1]. On utilise pour cela l'absorption de l'énergie des photons par effet Compton : le détecteur double est formé de deux segments électriquement indépendants, placés successivement sur la trajectoire des photons ( fig. 1).

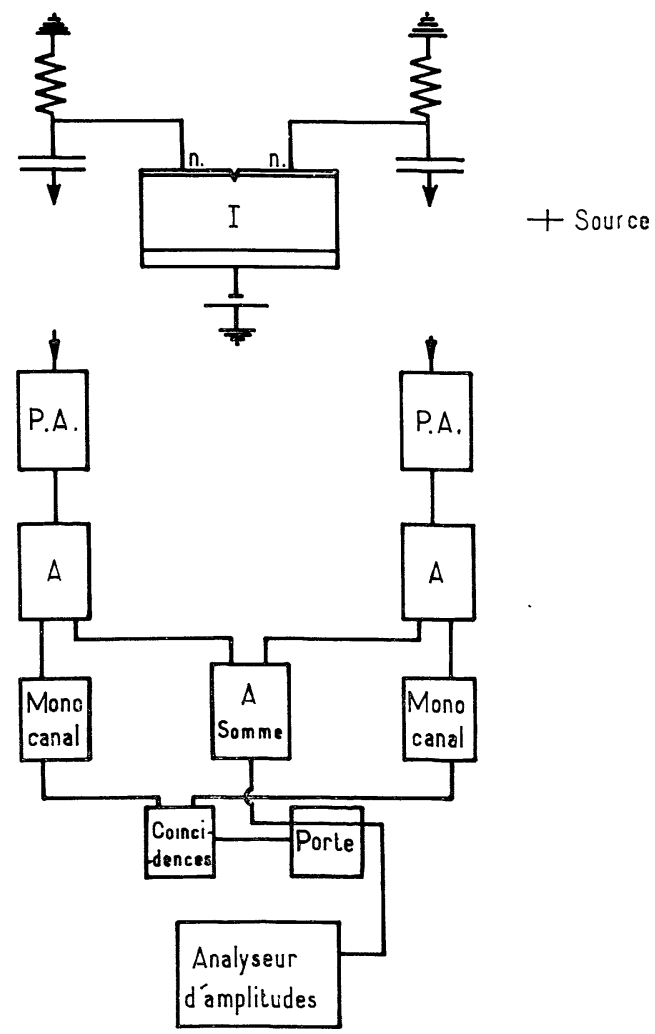

FIG. 1.
Le premier segment est chargé de détecter les photons rétrodiffusés par effet Compton, le second les électrons Compton qui leur sont associés. La somme de l'énergie de l'électron et du photon diffusés (détectés simultanément) est effectuée et correspond à l'énergie totale du photon incident.

I. Fabrication du détecteur. - Nous avons fabriqué une jonction à partir d'un lingot de germanium dont la résistivité était de $29 \Omega / \mathrm{cm}$ (surface $20 \mathrm{~mm} \times 20 \mathrm{~mm}$, épaisseur diffusée $7,5 \mathrm{~mm}$ ). La zone intrinsèque était située à environ $320 \mu \mathrm{m}$ de la surface. Nous avons alors, d'un trait de scie dont la profondeur était de $400 \mu \mathrm{m}$, séparé la zone $\mathrm{N}$ en deux parties sensiblement égales. Les deux segments contigus ainsi réalisés étaient électriquement indépendants, et nous n'avons observé par la suite aucune réinjection de l'une à l'autre. Ce double détecteur a été placé dans un cryostat classique à vide statique refroidi à l'azote liquide, les photons pénétrant par une face latérale parallèle au trait de scie.

II. Montage électronique ( fig. 1). - Les détecteurs, polarisés à $1500 \mathrm{~V}$ par une source unique de tension, délivrent des impulsions amplifiées par des chaînes dont les gains sont égaux à mieux de $0,5 \%$. Le sélecteur de coïncidences placé à la suite des sélecteurs d'amplitude monocanaux a un temps de résolution de $0,2 \mu s$.

III. Résultats. - Le sélecteur d'amplitude analysant les photons diffusés a été calé dans la bande d'énergie située entre $160 \mathrm{keV}$ et $230 \mathrm{keV}$, le sélecteur analysant les électrons Compton entre $250 \mathrm{keV}$ et $1500 \mathrm{keV}$. Nous pouvons de cette façon analyser les photons gamma subissant une rétrodiffusion à $180^{\circ}$ dont l'énergie est située entre $420 \mathrm{keV}$ et $2000 \mathrm{keV}$. La résolution en énergie (largeur à mi-hauteur) est de $4,6 \mathrm{keV}$ à $1,28 \mathrm{MeV}$. Les figures 2 et 2 bis montrent 
les spectres obtenus pour une source émettant des photons de $0,511,0,660,1,170,1,280$ et $1,330 \mathrm{MeV}$ en acceptant tous les événements d'une part, en utili-

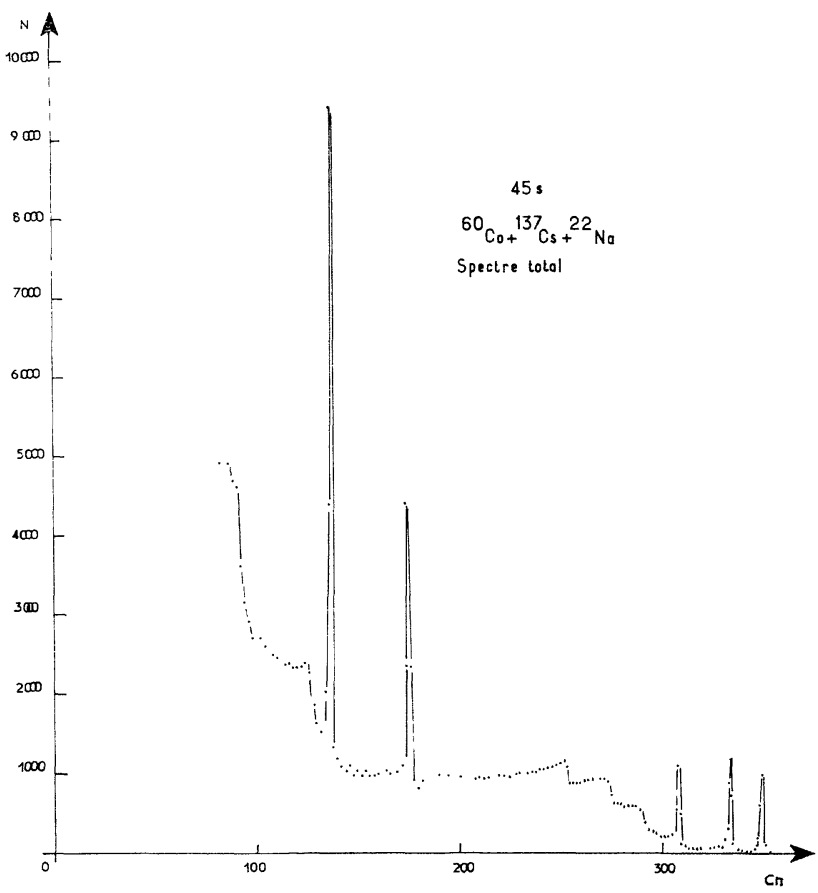

FIG. 2. sant le dispositif Compton d'autre part. On constate que la surface des paliers Compton a été réduite d'un facteur supérieur à 10 .

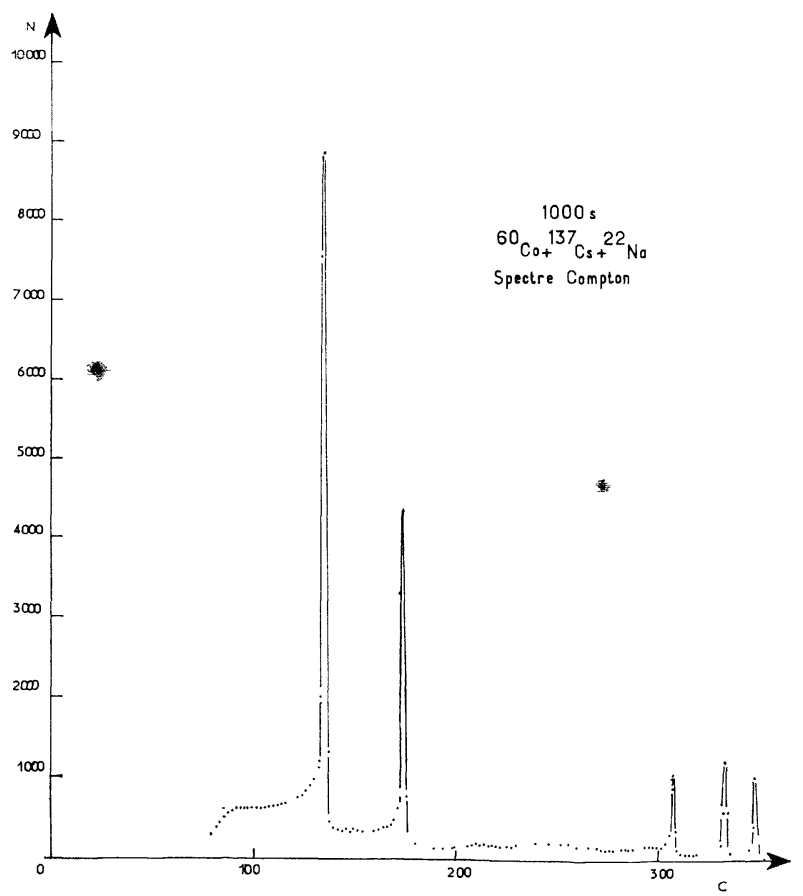

Fiv. 2 is

BIBLIOGRAPHIE

[1]. GRUHL, Nucl. Instr. Methods, 1967, 54, 2. 